\title{
Optimization and Simulation of a Dynamic Management System for Building Construction Based on Low-Power Wireless Sensor Networks
}

\author{
Ning Qi $\mathbb{B}$ \\ School of Architecture and Design, Changchun Institute of Technology, Jilin Changchun 130021, China \\ Correspondence should be addressed to Ning Qi; jz_qn@ccit.edu.cn
}

Received 10 November 2021; Revised 18 January 2022; Accepted 22 January 2022; Published 9 February 2022

Academic Editor: Gengxin Sun

Copyright (c) 2022 Ning Qi. This is an open access article distributed under the Creative Commons Attribution License, which permits unrestricted use, distribution, and reproduction in any medium, provided the original work is properly cited.

\begin{abstract}
In this paper, we use a low-power wireless sensor network to conduct in-depth research and analysis on the optimization simulation of the dynamic management system of building construction and study the specific application of BIM technology in the information management of building construction sites. By analysing the classical delustering routing and combining the advantages in the delustering routing protocols, we propose a two-level Mesh wireless sensor network delustering routing protocol in terms of cluster head uniformity, energy consumption and balance, working mode, and handling of isolated nodes. The most important feature of this protocol compared to other delustering protocols is that it retains the design of flat network topology and designs three routing methods with the corresponding functions of wireless sensor networks to achieve better network performance. The supported personnel location technology and construction measures and environmental monitoring technology are introduced, the applicability of the technology in the building construction process is compared and analysed, the topology of construction measures and environmental monitoring technology is designed, and finally, the targeted development of the collection scheme for personnel, scaffolding, formwork, pits, cranes, construction hoists, and physical environment is completed. Firstly, it analyses the development status of BIM-based building construction site information management technology and application and discusses the basis of BIM technology implementation and the advantages of BIM technology application in information management. Then, this paper constructs the framework of a BIMbased building construction site information management system from three dimensions: functional, logical, and physical. To ensure the realizability and operability of the system, this paper discusses the operation mechanism of the BIM-based building construction site information management system, studies the key technology of system operation, designs the basic operation process of each application system of the system, and elaborates the organizational structure of the building construction site and the functional division of the relevant personnel.
\end{abstract}

\section{Introduction}

The construction industry is a labour-intensive industry; its operating environment is complex; there are more unsafe factors; the volume of the production of construction products is large; the operating time is long; there are many types of safety accidents at construction sites; and falls from height, object strikes, mechanical injuries, collapses, and electric shock are the five major injury accidents in the construction industry. Falls from height are the most frequent type of accident, accounting for $53 \%$ of accidents, followed by object strikes. The probability of safety accidents becomes greater because safety managers alone cannot detect safety hazards and solve them in time by relying on on-site inspections, while the probability of safety accidents becomes greater due to the rapid renewal of personnel in the construction industry and low safety awareness of personnel and formal safety education of workers by managers [1]. Competition will inevitably occur, resulting in the failure of some of the data to be sent, and the data must be sent through multiple retransmissions. Construction safety accidents not only bring huge loss of life and property to society, enterprises, individuals, and their families but also bring serious negative impacts to the image 
of enterprises and social stability. The economic losses caused by safety accidents include not only the compensation costs for the families of the accident victims, the rescue and medical costs, and the costs of accident penalties but also the losses caused by the stoppage of work due to the accident and the losses caused by the replacement of workers [2]. The low level of attention to technological innovation in construction technology and management and the low investment in technological research has left the development of construction technology in a stagnant state [3]. Most of the construction machinery and safety protection used in construction currently have no essential improvements, and the way of safety management is almost indistinguishable from before, leading to a lack of safety control on site. The use of emerging technologies in the construction industry can shift the focus of safety management to the prior, to achieve preventive management, based on emerging technologies of safety warning management can not only monitor in real-time human behaviour and the state of things the identification of unsafe factors in construction and early warning but also serve as a platform for safety management, to support other safety management-related work content, such as safety education, safety training, and safety records.

Safety is not just an issue for the construction industry but also an issue that needs to be a key concern for the whole society. Modern information technology is developing at a high speed, bringing immeasurable impact to the construction industry, so construction safety management needs the information technology industry to assist and to develop a clear strategic plan when the country builds a construction safety system. This paper delves into the application of construction safety management based on BIM technology, exploring how the use of building information modelling can make the construction safety management level improve, prevent and reasonably control the safety accidents that occur with the construction site in advance, and promote the construction industry to the road of sustainable development [4]. The construction industry is in a state of rapid development, and the characteristics of modern buildings are reflected in the large volume, large span, complex structure, and deep foundation, resulting in a higher accident rate in the construction stage. BIM technology applied in the construction stage can effectively find out the safety hazards and danger sources in construction management and technology and deal with the construction safety hazards through simulation [5].This paper deeply analyses BIM technology application management and engineering safety management, which makes it possible to further improve the relevant safety management procedures; improve and adjust safety programs and safety plans several times which can make the rate of safety accidents before and during construction decrease; meet the national construction development policy; promote construction informatization; strengthen the information communication between building designers, owners, and construction, etc.;and reduce the cost of resources invested. Information technology should be used throughout the whole period of construction and maintenance.
Due to the complex and variable operating environment faced by the passive sensing nodes, the magnitude of the input signal faced by the power amplifier is not stable and constant. In this case, the overall efficiency of the amplifier over the entire input signal power range becomes more important than the optimal efficiency. To improve the overall amplifier efficiency, this paper proposes a method for input RF power monitoring and intelligent control of the bias voltage. Based on ensuring that the amplifier itself has a high peak efficiency, its efficiency in the case of a small signal input is improved by intelligent bias control techniques. WSNs are typically task-based networks with the data collected by all nodes in the network as the core, and WSNs are generally said to be data-centric networks. WSN can also serve as a good backbone network in the monitoring area under the condition of restricted communication methods, and if it can be converged with other networks, it can carry more functions and enhance the flexibility of WSN. The construction personnel and management personnel on the construction site need a lot of construction information to support the construction activities. The use of a protocol conversion interconnection scheme is more convenient; you need to complete the protocol conversion between the interconnection nodes while providing the appropriate support in the backbone network protocol. Therefore, you can let the WSN in the sensing nodes as interconnection nodes to complete the protocol conversion, while designing the corresponding WSN routing protocol. In terms of protocol design, the focus should be on adding network convergence support and extending the network lifetime of WSNs. With the network fusion of WSN as the research objective and the optimization direction of improving the energy usage efficiency, the WSN routing protocols applicable in different applications are proposed. Through protocol design and optimization, simulation testing, and physical testing, the convergence of WSNs with individual domain networks is achieved. This paper tentatively explores the field of WSN network convergence and further verifies the feasibility of WSN network convergence through hardware platforms in addition to simulation and explores the potential applications of WSN as a backbone network for monitoring areas.

\section{Related Works}

Among the many technical difficulties of wireless sensor networks, the most important one is the characteristic of their limited energy supply. In recent years, wireless sensor networks have been facing many challenging problems in the process of rapid development, among which, energy consumption is the core issue, which is one of the main differences between wireless sensor networks and traditional wireless networks [6]. Traditional wireless networks are mainly focused on meeting the increasing requirements of users for network service quality, and the energy of its network equipment terminals can be replenished, and the energy consumption is not the main issue that restricts the development of network technology. In contrast, due to the 
limited energy supply of the nodes, the energy of the nodes becomes the biggest factor limiting their continued development [7]. Once the node energy is exhausted, the node will stop working immediately. To be able to extend the life of the node, it is necessary to improve the efficiency of the entire node for energy utilization. The battery is the core of providing energy to the node; however, the technology level of the battery is relatively slow to develop, and in the past long time, the energy density of the battery has improved to a very limited level [8]. Therefore, in addition to using environmental energy such as solar energy, mechanical energy, wind energy, electromagnetic energy, vibration energy, and temperature difference energy to supplement the node battery energy, so that the node will achieve a certain degree of self-power, the main way is to reduce the energy consumption of wireless sensing nodes or wireless sensing nodes for low-power design [9]. Therefore, research on low-power technologies for nodes in wireless sensing networks is important to promote the level of performance of wireless sensing networks revealing that the chain reaction of factors with cause-and-effect relationships is the basic process that causes accidents and has a great impact on safety management [10].

Scholars are more advanced in their research on safety management and have developed visual $4 \mathrm{D}$ technology models to link personnel in various departments thereby increasing team safety awareness. Traditional construction safety management systems and practices are analysed, and a cloud-based safety information and communication system is designed and developed using a free online web server with features including preinitiation safety meeting minutes, access requests and approvals, job safety analysis, and safety incident reporting, and prospects for cloud computing in construction safety management are indicated [11]. At the same time, the transmission is fast and the information fidelity is high. However, due to the need for cables to be laid in the monitoring area, it is difficult to arrange the monitoring sites with complex environments. At the same time, maintenance during the later period is also difficult. The idea of establishing a set of construction safety management systems based on China's national conditions is proposed, and how to do a good job of construction safety management in China is analysed from the legal level, political level, economic level, and cultural level. Some scholars believe that the development of China's construction safety production management is not stable enough, the safety management responsibilities are not clear, and there is a lack of scientific basis and favourable theoretical guidance in construction safety production management; the main defects of the construction safety production regulation system are that the legal normative documents do not play a full role, the laws and regulations supporting the formulation of slow laws and regulations are too principled and not strong in science, and the entire regulation system is still lacking in content [12].

The government should improve safety legislation and strengthen safety law enforcement, based on the focus on highlighting the service guidance function, and complemented by the role of market mechanisms to guide construction enterprises to consciously pay attention to safety production. The process of improving the efficiency of safety management should pay attention to the importance of construction safety management personnel training mechanism and also needs to improve the supporting mechanism for the development of safety management personnel in construction enterprises; some scholars from universities, enterprises, and government form three levels of in-depth analysis of countermeasures and measures to train safety management personnel and to provide analysis and reference for the establishment of domestic construction safety management personnel training mechanism. Wireless sensing in the disaster site monitoring data collection applications is also very extensive at present; it can achieve not only early warning of landslide geological disasters but also wireless sensing network monitoring system, deployed in areas prone to secondary disasters, to assist in the implementation of search and rescue tasks, to improve the safety and reliability of rescue.

\section{Simulation Analysis for Optimization of Dynamic Management System for Low- Power Wireless Sensor Network Building Construction}

3.1. Design of a Dynamic Management System for LowPower Wireless Sensor Network Building Construction. Construction safety early warning data information collection is the key problem that needs to be solved for the whole early warning system, and only if the data has authenticity and timeliness can the practicality of the whole early warning be guaranteed [13]. Data information collection in the traditional way is obtained through human inspection or measuring instruments, which not only consumes a lot of workforce and material resources but also has limitations and errors in the data obtained, which cannot meet the needs of managers for first-hand data. With the progress of science and technology, information technology is developing rapidly, and wireless sensor network technology has brought a breakthrough to data collection, which can not only meet the various needs of data acquisition but also preprocess and analyse the data.

$$
\begin{gathered}
R Q=k_{1} \cdot \text { Hop }-k_{2} \cdot\left(\alpha_{1} \cdot R S S I_{\max }-\alpha_{2} \cdot L Q I_{\text {max }}+\alpha_{3} \cdot U I_{\text {max }}\right), \\
\text { Hop }=\left(1+\frac{\text { Hopnum }+1}{\text { Hopnum }_{\text {min }}}\right) \times 256 .
\end{gathered}
$$

Wireless sensor network (WSN) technology refers to a distributed sensing network composed of various types of sensor nodes with physical information collection functions, data processing functions, and wireless communication functions. The various types of small sensing devices at the end of the sensing network are small but fully equipped with sensing devices, embedded microprocessors, and radio transceivers, so they can not only sense any relevant physical information but also have sensing capabilities, data 
processing, and communication capabilities for automatic transmission to wireless base stations [14]. This can speed up the progress of construction. Therefore, the level of construction technology is also a key factor in determining the construction progress. Thanks to developments in the field of microelectronics and wireless communication technologies, miniature, inexpensive, and intelligent sensors are deployed in physically monitored areas through wireless connections, offering great convenience over the previous wired connections. Wireless sensor network technology is now widely used in various fields, and it is bound to become a bridge between the physical world and the computer world and will revolutionize the way we live, work, and interact with the physical world.

$$
\begin{gathered}
A M P R=\frac{\sum_{i=1}^{M} \max P w r_{i}^{2}}{N}, \\
C H_{\text {prob }}=\min \left(C_{\text {prob }} \cdot \frac{E_{\text {residual }}^{2}}{E_{\text {max }}}, P_{\text {min }}\right) .
\end{gathered}
$$

The design of wireless sensing networks is mainly applied in harsher environments, and the number of nodes required is generally much more than that of traditional communication systems, which are high-density wireless sensing networks. The sensing nodes in wireless sensing networks not only take the responsibility of collecting data but also take the responsibility of receiving data transmitted by other nodes. The networking method of wireless sensing networks is self-organization and multihop etc., which is more flexible. Self-organization is equivalent to plug-andplay, while the topology will be affected by the joining or withdrawal of sensing nodes, which belong to the dynamic topology. If the sensing nodes move, the topology is also updated according to the relative position of the sensor nodes. This also ensures the stability of the system; when one or more of the sensing nodes in the sensing network fails, it does not lead to stagnation of the entire sensing network system [15]. The sensing network is divided into wired sensing networks and wireless sensing networks. A wired sensing network establishes a channel for information transmission through a line connection, which is highly resistant to interference, and the information transmission process is less affected by the outside world. Wireless sensing networks can carry out information transfer between nodes through a wireless network and aggregate to the gateway for conversion to achieve the self-organized connection between the wireless network and wired network, as shown in Figure 1.

The sensor node layer is composed of a data acquisition hardware unit, a processing control hardware unit, a communication hardware unit, and an energy hardware unit that implements the data acquisition, preprocessing storage, and transmission functions of the sensor nodes. The sensor node layer is implemented by arranging many sensors in the monitoring area, thus requiring the sensors to provide as much power as possible with minimum expense, minimum size, minimum weight, and maximum lifetime.

$$
C I=w_{1}^{2} \frac{E}{E_{\max }^{w}}-w_{2} \frac{C}{C_{\min }} .
$$

The topology discovery request sent by the aggregation node and the broadcast sent by the cluster head election completion use the cluster head broadcast; the cluster head broadcast contains the hop count and the information used to calculate the route quality; the hop count is used to limit the range of the broadcast; and other sensing nodes in the cluster can calculate the route quality and establish the route to the cluster head based on the data in the message. There is no safety problem; otherwise, it is necessary to warn the safety management personnel to check the relevant mechanical equipment and arrange maintenance personnel to repair. The cluster head sends a reelection broadcast message when it is reelected; the node receives it, calculates the cluster head index using the route control message, and notifies the cluster head node with a node election information reply message. The cluster head node elects a new cluster head based on the cluster head index of the nodes in the cluster and notifies the node with a cluster head election confirmation message; upon receipt of the notification, the node sends a cluster head broadcast message; if the previous cluster head receives the message, then the election is successful; if not, then it is sent several times. If the previous cluster head does not receive the cluster head broadcast message several times, then this election fails and the cluster head election is conducted again. Additional information about the transport method, the list of transport methods, and the status of the route are added to the four base messages in the previous chapter. The transmission method indicates whether the route is a long-range route or a close-range route. The aggregation node can be regarded as an intermediate gateway node connecting the sensing network and the extranet, realizing the conversion and interoperability between different protocols, and the data information from the sensing node is delivered to the aggregation node using a single-hop or multihop approaches [16].

The convergence node layer is in the middle layer between the sensing node layer and the management node layer and plays a role in the organizational structure to carry on the task to the sensing node layer according to the requirements of the management node, whose physical structure is essentially equivalent to the enhanced function of the sensing node. At the same time, the aggregation node is also responsible for the establishment of the network and the joining or withdrawing of sensors in the sensing network. The management node layer is in the uppermost layer of the whole wireless sensing network and its authority level is the highest; the user can use the management node to achieve information resource access to the wireless sensing network system; if the user needs to change the settings in the wireless sensing network, it also operated through this layer, as shown in Figure 2. It accounted for 53\%, followed by object strikes. Simply relying on safety management personnel to patrol the site cannot find and solve potential safety hazards in a timely manner. 


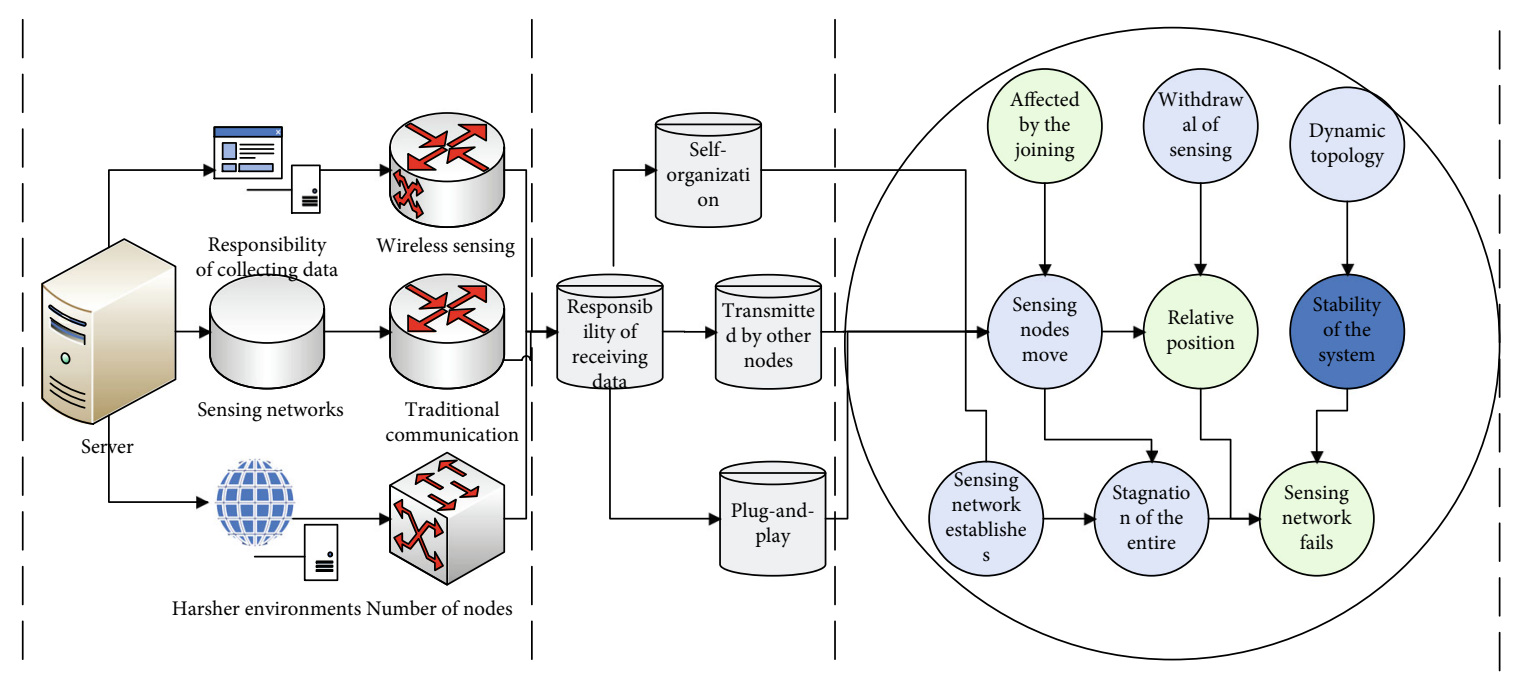

FIGURE 1: WSN three-level network structure.

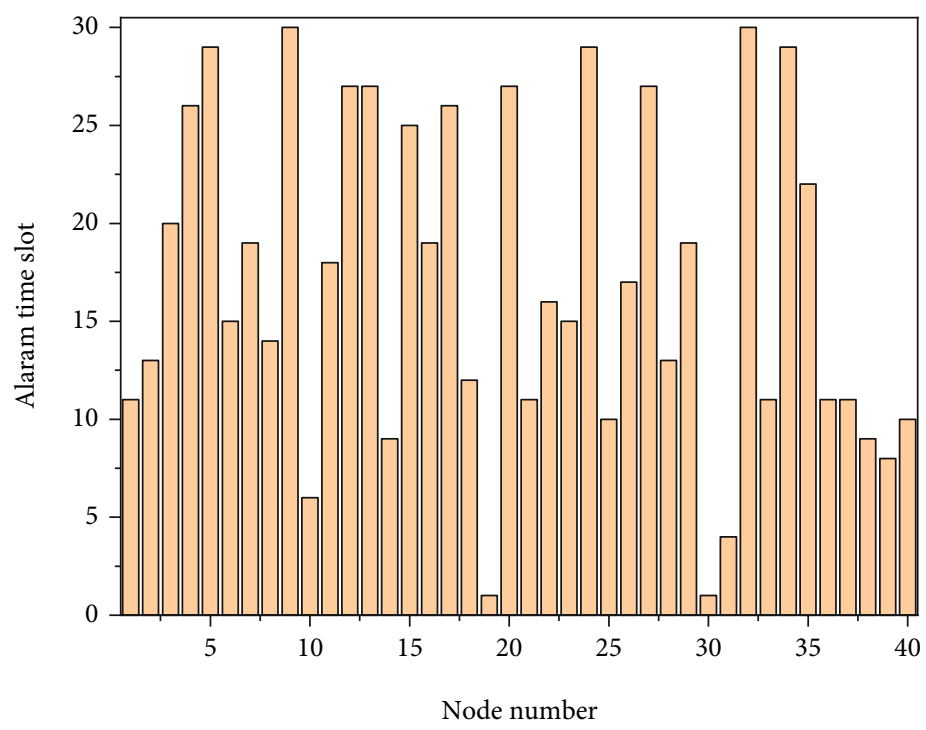

FIGURE 2: Node alarm time slot.

When multiple nodes are ready to send data at the same time, contention will inevitably occur as they go to occupy the transmit channel at the same time, failing to send some of the data and having to finish sending the data by multiple retransmissions [17]. The collision of data not only causes a delay in the working of the whole system but also increases the extra power consumption and increases the energy consumption of the whole system due to multiple retransmissions. Wireless channels have broadcast characteristics; when a node sends a message, the nodes within the entire network can receive the information it sends. For nondestination nodes, receiving these messages that are not valid for themselves not only consumes extra energy but also may disrupt their own. During wireless communication, nodes have four states, which are sleep, listening, receiving, and sending.

$$
w_{1}-w_{2}=1
$$

Among them, the sleep state has the least power consumption, and the power consumption for sending data and receiving data is relatively high. When in the listening state, the node constantly listens to the wireless channel to determine whether the channel is idle or not. Since wireless sensor networks are relatively small data networks, it is not necessary for the nodes to always listen to the channel, and they can completely go into a dormant state when there is no task. The design concept of WSN is data-centric. Data from all nodes are transmitted to the aggregation node. Compensation expenses, rescue and medical expenses, accident penalty expenses, etc., also include the loss of shutdown and rectification caused by the accident and the loss caused by the replacement of workers. The user is 
mainly concerned with monitoring the data collected by the sensing nodes in the area, and the protocol design needs to consider the management and processing of the data. With limited energy, how to minimize energy consumption and increase the network life cycle while discovering routes and transmitting data is the focus of the routing protocol design.

The communication between sensing nodes is improved on the original communication flow. The communication flow finds the proximity route first when discovering the route and then further discovers the distance route if there is no proximity route for the destination node. The advantage of this is that when the nodes are close to each other, only a small amount of network and energy resources are consumed to establish a route to communicate with each other. For distant nodes, the additional consumption in finding proximity routes is only a very small part of the complete route-finding process and has less impact on network lifetime and network performance.

\subsection{Simulation Analysis of Construction Dynamic} Management System Optimization. Information refers to news, intelligence, knowledge, etc. transmitted (conveyed, transmitted) by oral, electronic voice, mail, or other relevant written means, usually manifested as sound, text, figures, and images, etc. In the field of engineering project management science, a large amount of engineering information is formed with the flow of workflow, coordination, capital flow, etc. in the process of project implementation, which are processed and formed into specific forms of data to provide the basis for decisionmakers to control the project implementation process [18]. At the same time, the information flow also forms an interrelated whole of the project's capital flow, coordination, project organization, and various management functions, as well as the external project environment, which influences and controls the workflow, coordination, and capital flow. Only when the information flow is smooth can the project be implemented smoothly. The building construction site is the convergence place where various construction operations and construction activities are carried out and completed at the same time, and it is also the centralized place where building construction information is generated, transmitted, and applied. Construction personnel and managers at building construction sites need a large amount of construction information to support construction activities. To explore a new system of construction site information management, it is necessary to identify and categorize construction site information. The building construction site is the gathering place where the design results of different professions such as architecture, structure, HVAC, water supply, and drainage are transformed into the physical building, and it is also deeply influenced by the decisions of project planning, design, and construction. So, the construction site of the building construction information sources is wide, and the content is complex and changeable, and lack of on-site security controls. The use of emerging technologies in the construction industry can shift the focus of safety management to advance management and realize preventive management. Safety early warning management based on emerging technologies can not only monitor the behaviour of people and the state of things in real-time. Construction project construction lasts for a long time, with many uncertainty factors and a high frequency of information changes [19]. Construction site engineering information is also updated in real-time with the progress of the project, and the updated engineering information can be used as the next stage of information. Therefore, the engineering information is interdependent and transforms and influences relationships, which often involves the whole and is highly correlated, as shown in Figure 3.

In general, on-site information can be divided into structured and unstructured information. Structured information refers to information data that is stored and described by open data standards, and such information data can form multiple components with clear hierarchical structure and interconnection. In the application of BIM technology in building construction sites, structured information can be searched quickly by determining its characteristic nodes, which has the advantages of improving information utilization rate, completing information search efficiently, and reducing missing information; unstructured information refers to information data with relatively unclear form, poor correlation, and blindness when querying information, and at present, it mainly relies on manual means to modify and update and organize, such as electronic document reports, e-mails, web pages, video files, and BIM model information of nonpublic data standards. Wireless monitoring technology for construction measures and environment can be classified into two categories according to the transmission method; one is the wired transmission method.

Because the data information is transmitted by way of cable, it is less interfered with by the outside world, while the transmission is faster and the information fidelity is high, but because the cable needs to be laid in the monitoring area, the arrangement is more difficult for the monitoring place with a complex environment, while a certain degree of difficulty also exists with the later maintenance in the process of use.

Especially on the construction site, many cables lying about will affect the normal construction of the operator, while the operator might easily to destroy the cable during the construction process. Prevent and reasonably control the safety accidents on the construction site in advance, and promote the sustainable development of the construction industry. The construction industry is in a state of rapid development, and modern buildings are characterized by large volumes, large spans, complex modeling structures, and deep foundations. The other type is the wireless transmission method; the wireless transmission method is transmitted by wireless signal; the signal carries monitoring information, relying on the current rapid development of wireless transmission information technology, and can be applied to the monitoring of harsh environments; therefore, the wireless transmission method has been favoured by the field of structural monitoring and 


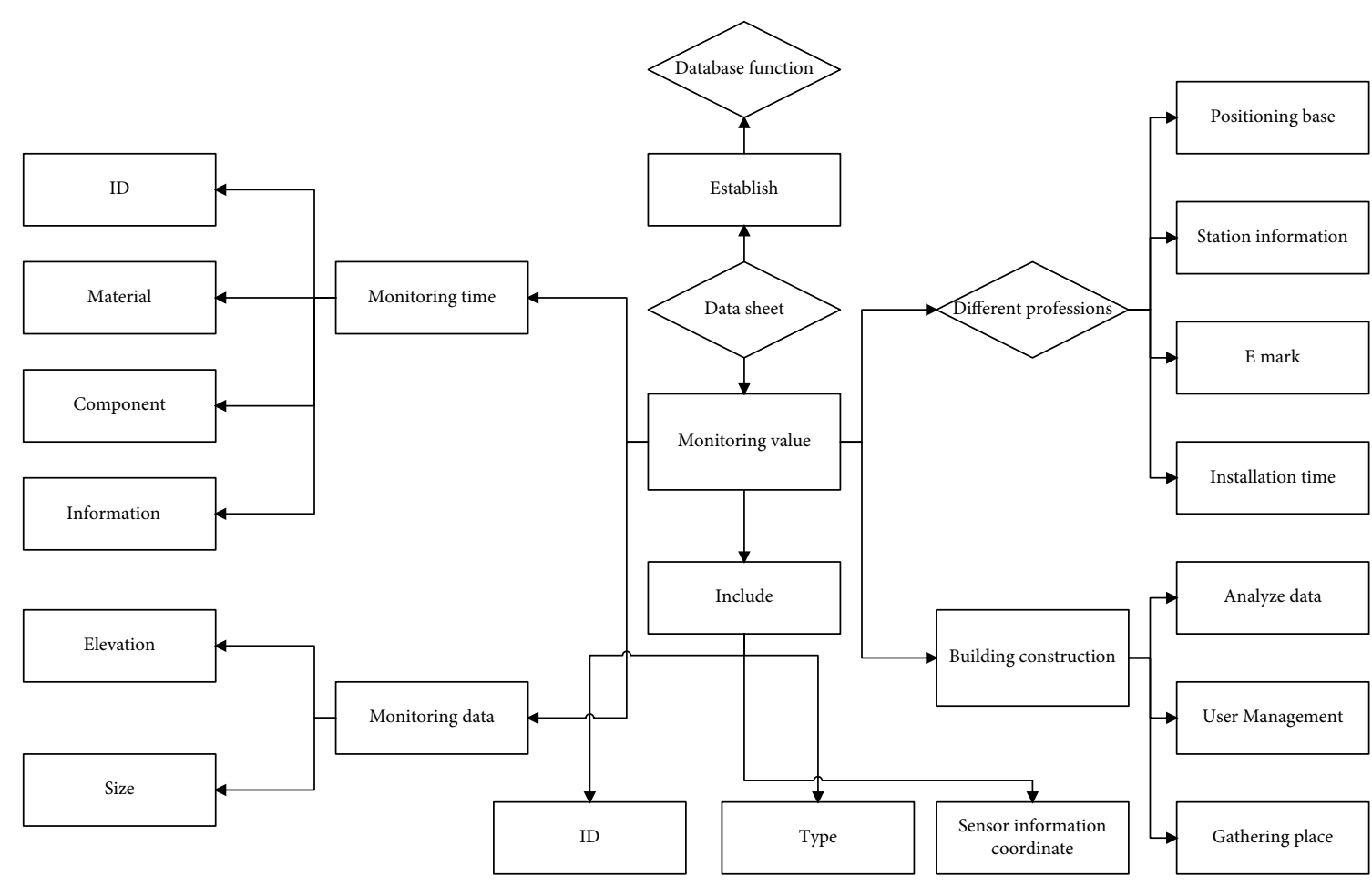

FIgURE 3: Monitoring information database ER diagram.

is slowly replacing the wired transmission method. The research on the construction methods of digital terrain and 3D hydraulic building models is an important part of the research on dynamic 3D total view management methods for construction, and this section will carry out the research on digital terrain construction methods, 3D model construction methods, and ground coupling methods based on BIM ideas and combined with the characteristics of hydraulic construction modelling.

There are many components in the construction of the sea trial terminal, the construction process is complex, the volume of work is large, and the duration is long. In the actual construction process, it is easy to have factors affecting the construction schedule, such as problems in the supply of construction materials during the construction process, unexpected weather, typhoons, and rainstorms encountered during the construction process; an insufficient number of construction personnel will lead to delays in the construction schedule. Construction schedule management generally refers to the analysis of relevant influencing factors and making reasonable adjustments, using certain measures to achieve the project schedule adjustment optimization, as shown in Figure 4.

The influencing factors of construction schedule and construction schedule optimization methods are closely related, and the constraints of the construction optimization model can be determined by investigating and analysing the influencing factors of the construction schedule. Resources such as workforce, material supply, and quantity of machinery and equipment have a great influence on the construction progress; if the resources are insufficient, the construction progress will be delayed because of the inability to increase the construction input; on the contrary, if the construction resources are in excess and greater than the construction capacity, it will cause the waste of resources, thus increasing the construction cost and management difficulty. Sufficient funds are the guarantee that the project can be carried out smoothly, and in the case of sufficient funds, the project can be reasonably accelerated. Construction costs are composed of direct costs and indirect costs, and direct costs include labour, materials, and machinery, while indirect costs include management and site expenses. Therefore, it can not only sense any relevant physical information but also have sensing capabilities, data processing and communication capabilities and can automatically transmit to wireless base stations. Due to the development in the field of microelectronics and wireless communication technology, tiny, inexpensive, and intelligent sensors are deployed in the physical monitoring area through wireless connection. Therefore, the level of construction technology is also a key factor in determining the construction schedule.

In this regard, the system will define the structure of data and composes an Extensible Markup Language (XML) schema to achieve structured information storage; similarly, a special unstructured data architecture will be established to centralize the storage and management of unstructured data. In the whole process of building construction site information management, the system will establish different BIM database tables to record information metadata according to information categories and formats, thus forming a complete BIM-based data storage [20]. The IFC standard is the de facto data description standard of BIM technology, and most of the BIM professional application 


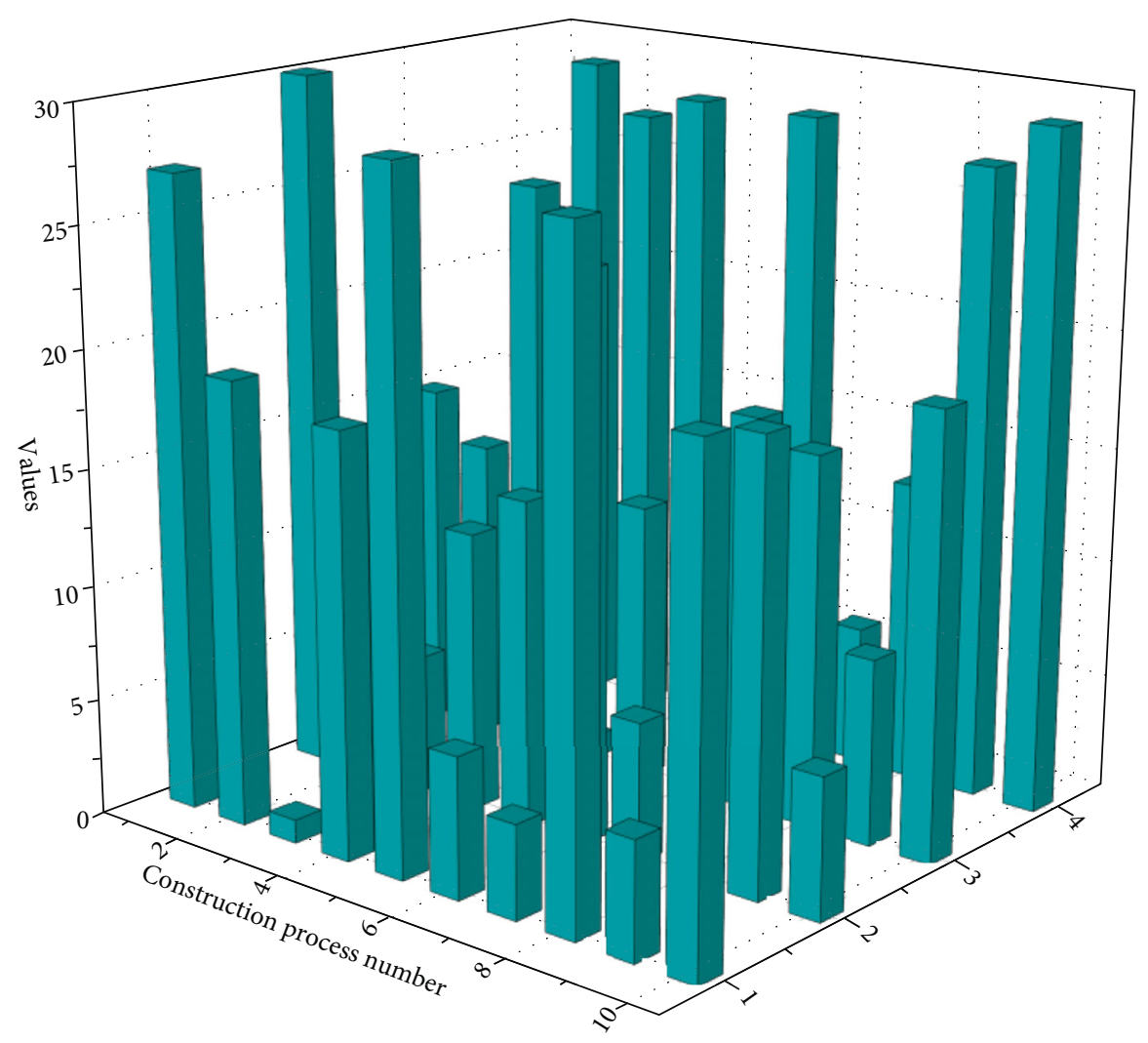

FIGURE 4: Construction schedule and costs.

software can support the input and output of the IFC building information model.

Through this module, the information manager issues instructions related to material and equipment management; the relevant personnel view the instruction information and generate the instruction feedback information; when the difference with the BIM data exceeds a certain area, the difference part is highlighted in the BIM model and the alarm information is quickly submitted to the front-end system and the information manager, while the module can be configured by the user to respond to the alarm linkage option according to the actual demand. In the process of BIM-based construction site information management, the information manager can implement comprehensive BIM-based information management of the quality, quantity, and use plan of materials and equipment at the construction site through the system, while nonconstruction site information managers such as material and equipment suppliers, equipment leases, and supervisors can only view and feedback the instruction information or make inquiries about contracts, inventory and other information.

\section{Results and Analysis}

4.1. Performance of Dynamic Management System for LowPower Wireless Sensor Network Building Construction. According to the data in Table 1, it can be learned that when the transmission distance is farther, the received power is lower. When the transmission distance from the receiving antenna to the transmitting antenna is $10 \mathrm{~m}$, the received wireless energy is $-18 \mathrm{dBm}$; when the transmission distance is more than $20 \mathrm{~m}$, the wireless energy received by the antenna is less than $-20 \mathrm{dBm}$. When the received infinite energy is lower than $-20 \mathrm{dBm}$, the converted DC energy is less efficient and difficult to collect. Therefore, in the simulated system, due to the limitation of the RF source transmitting power, the effective wireless transmission distance is limited to $20 \mathrm{~m}$, while in the actual shed application environment, the effective system receiving distance can be extended by increasing the RF source transmitting power.

From a theoretical point of view, the relationship between the effective transmission distance of a directional wireless transmission system and the transmitting power of the RF source is analysed. In this section, however, the focus will be on flexible receiving antennas that can be closely integrated with the greenhouse agricultural environment. Flexible antennas, usually nonplanar antennas that conform to the shape of a specific object, have been used as part of the surface of moving objects such as aircraft. Due to its conformal characteristics, it can be well applied under some nonregular objects or special application scenarios, satisfying the characteristics of concealment and not easy to destroy, and in recent years, conformal antennas have gradually become a hot spot for research. It is a dynamic topology. If the sensor nodes move, the topology is also updated based on the relative positions of the sensor nodes. As a high gain circularly polarized array world is used as the RF transmitting antenna in this paper, to avoid the polarization mismatch problem, the receiving antenna should also 
TABLe 1: Analysis of the wireless energy transmission link.

\begin{tabular}{lcc}
\hline Input signal power & Output signal power & PAE \\
\hline 8 & 22.3 & 10.7 \\
9 & 39.2 & 41.2 \\
10 & 16 & 30 \\
11 & 15.8 & 46.6 \\
\hline
\end{tabular}

conform to the characteristics of circular polarization. A circularly polarized rectifier antenna is proposed, which is made of flexible material and can be applied on cylindrical surfaces such as pipes to achieve a conformal effect and provide power to wireless sensing nodes. This section will mainly discuss the structure and simulation results of the rectified antenna.

The design of this paper includes three sensing nodes for data collection, while the relay nodes are in broadcast mode to continuously poll the data to send data requests to the slave nodes, and when the sensing nodes get the corresponding data requests, they send the data to the relay nodes. The conventional data communication is based on relay nodes, which are polled continuously through a single channel according to the sensor node number, and the polled sensor nodes send data to the relay nodes, while the unpolled sensor nodes are in the sleep state, waiting for the polling signal. And when the sensing node collects monitoring data to reach the alert point, a second channel is opened for alarm data transmission in order not to crowd the channel with normal communication, as shown in Figure 5.

An envelope tracking power amplifier is a kind of dynamic bias amplifier, which synchronizes the bias voltage of the amplifier after detecting the amplitude and phase information of the input signal so that the amplifier can always work in the region of higher efficiency. The envelope tracking technique has high requirements for envelope tracking power supply, and according to the actual requirements of this system, it was decided to use the control role of the microcontroller to achieve the function of dynamic biasing based on the idea of envelope tracking. Since the bias voltage needs to change according to the input signal power, the input signal needs to be detected first, and since the detection process is not expected to have too much impact on the final output signal, a small portion of the signal needs to be separated by a power divider or coupling module for detection. The detector unit directly uses a circuit module composed of detector chips, and the MCU at the back end converts and processes the analogy signal generated by the detector circuit after the detector is completed to obtain the input signal power and then controls the DC voltage generation module at the back end to generate a suitable bias voltage to power the power amplifier according to the power magnitude value.

\section{System Optimization Simulation Results}

To read and judge the electronic tag information on the mechanical equipment using the fixed or portable method, the permission information of the mechanical equipment includes the annual inspection information, maintenance information, whether there are maintenance problems, etc. The implementation of this function is also using the $\mathrm{C \#}$ language nested If statement; for the annual inspection information, maintenance information, etc. which belong to the date and time information, it is impossible to directly calculate the date difference; it needs to be converted to seconds It needs to be converted into seconds and then calculated. As shown in Figure 6, it is the same way as the judgment of personnel authority identification, when all the return values are True after the execution of the nested. It proves that the machinery and equipment can be used normally, and there is no safety problem; otherwise, it is necessary to notify the safety management personnel to check the relevant problem machinery and equipment and arrange the maintenance personnel to carry out maintenance. After the inspection is completed, the label information in the label of the machinery and equipment needs to be updated, and the return value is True for the nested If statement execution structure identified by the authority, and the warning reminder ends, as shown in Figure 6.

The principle of this construction safety early warning system for the structural warning is to collect construction data from the site and compare it with the design safety values, to warn when the collected data exceeds the design range, and to consider the site in a safe construction state if it is within the design safety values. Therefore, the use of the early warning system requires the project department of the construction unit to establish good communication with the design unit and the design department of the construction unit, to carry out the design and safety technical briefing based on the construction safety early warning system, to accurately account for the initial value (safety design value) of this early warning system, and to improve the practicality of the safety early warning system.

This construction safety early warning system has four modules; considering the actual situation on-site and cost factors, the required module functions can be used selectively, and the monitoring of site personnel, machinery, structure, and physical environment can also be selectively installed and monitored according to the actual situation of the project; for example, the construction project undertaken by the construction unit has low floors, the external scaffolding is relatively simple to erect and the danger is minimal; considering the cost reasons, the structural monitoring of external scaffolding is not required, as shown in Figure 7.

The construction progress management module consists of two parts: two-dimensional construction progress management and three-dimensional construction progress management. The two-dimensional construction progress management function module is designed based on dynamic chart technology and database mining technology, which outputs the construction progress data optimized by the system as visual charts. The system's two-dimensional construction progress management generates the plan dynamically into a Gantt chart, which shows the total 


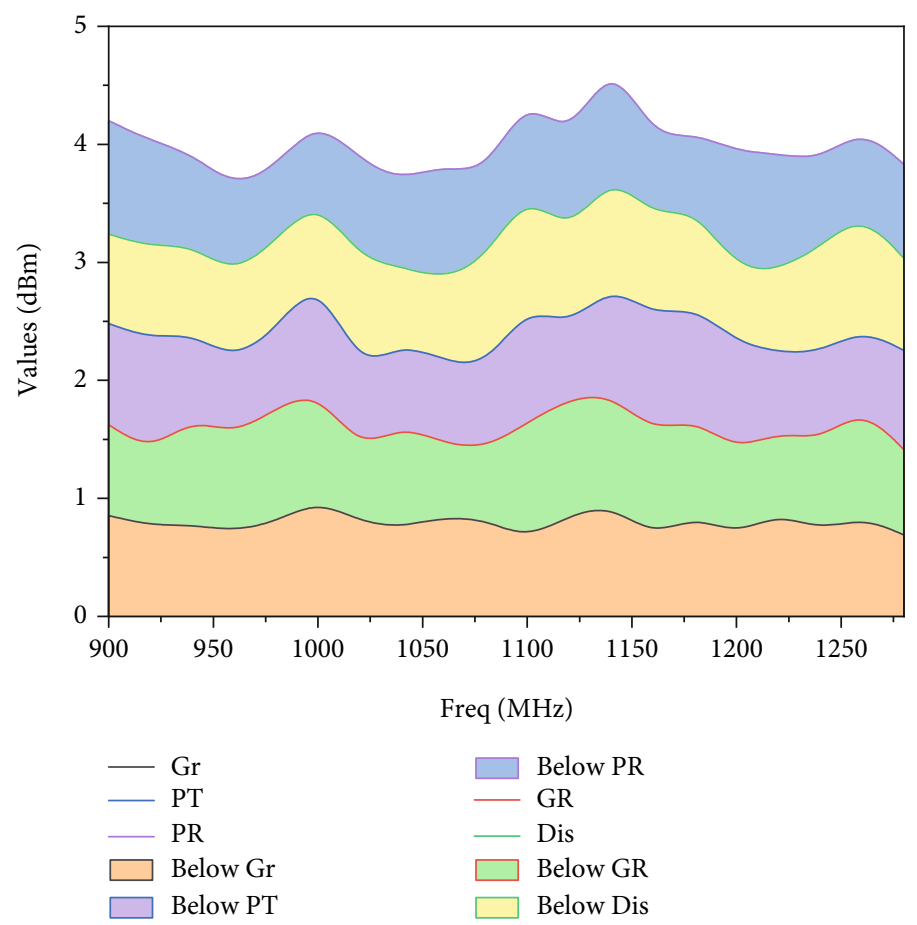

FIgURE 5: Output signal power-in, gain, and PAE test data plot.

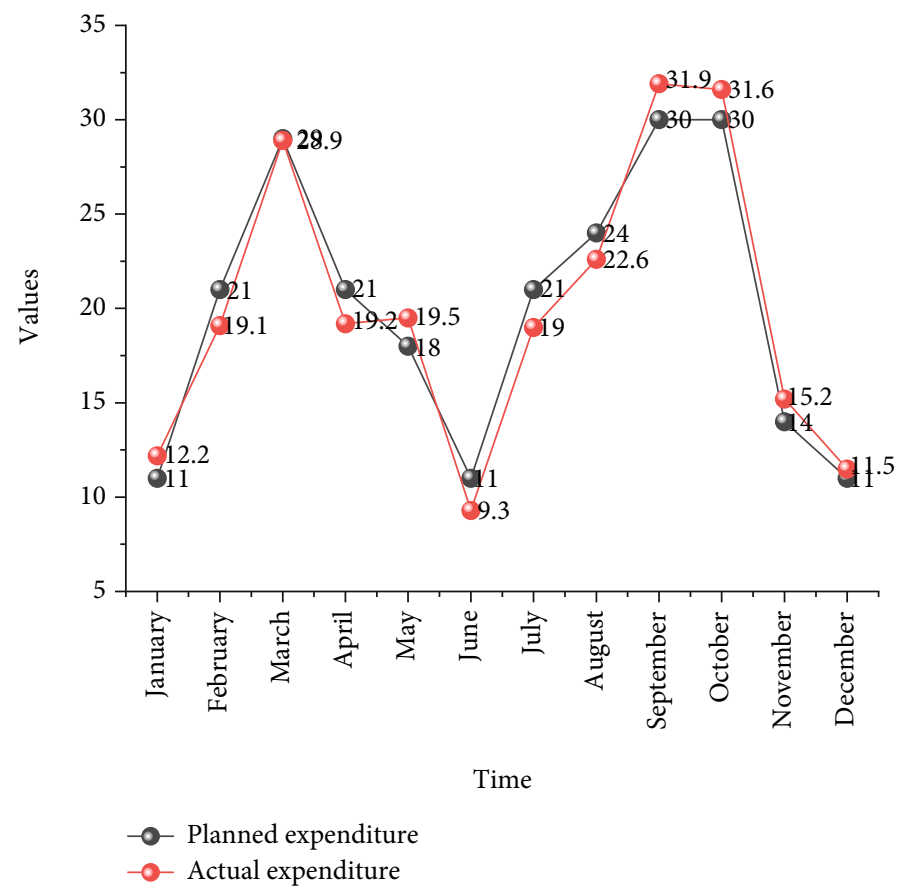

Figure 6: Use of funds and resources.

construction progress at the top and the unit project progress and schedule at the bottom, where the green part indicates that the section has completed construction and the red part indicates that the section is not yet under construction. After the node receives the notification, it will immediately send the cluster head broadcast message. If the previous cluster head receives the message, the election is successful, and if it does not receive the message, it will be sent multiple times. The left side of the construction progress Gantt chart shows the unit project progress plan attributes and the project progress display time zone; the unit project construction progress plan attributes include project progress, project start time, project duration, project end time, and the project name of the project; click on a unit project in the right side of the Gantt chart; the left-side attribute information column will display the corresponding 


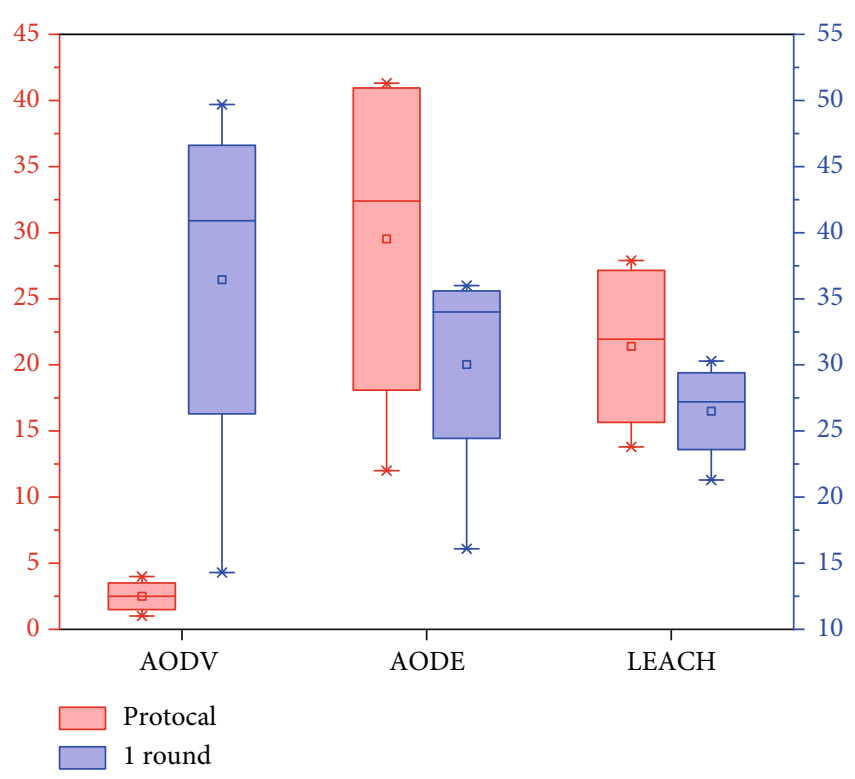

FIgURE 7: Protocol simulation results.

attribute information and the project progress display time zone. You can adjust the start and end time of the chart displayed on the right, and you can adjust the time unit of the construction progress Gantt chart display. Based on the above functions, the design of the two-dimensional construction progress management of the sea trial pier is completed. The spring-rectangular piezoelectric cantilever beam has a wide resonance range at the resonance point, and the bridge is in low-frequency vibration most of the time, so more energy can be collected by designing a piezoelectric energy harvesting device to integrate the triangle and springrectangular piezoelectric cantilever beam.

\section{Conclusion}

The collection of construction site data information is realized through WSN technology, including the information collection of people supported by WSN technology for personnel positioning technology; the information collected is mainly location information and attribute information; For the information collection of things supported by WSN technology for construction measures and environmental monitoring technology, the information collected mainly measures structure information, location information and environmental information. At the same time, comparative analysis and algorithm research on location technologies are conducted, topological network structure design for construction measures and environmental monitoring technologies is completed, and information collection schemes targeting people and objects at construction sites are developed. The design of the construction safety early warning system includes the determination of design principles, system framework design, initial value design, functional module design, process design, and use authority design. The construction safety early warning system mainly takes the site personnel of the construction unit as the monitoring object of the occurrence of safety accidents, and the use of this early warning system can realize the behaviour or state of reminding and informing people and objects of possible safety accidents and realize the functions of safety education, daily attendance management, and safety reward and punishment management. The physical framework of the system is then designed from the actual situation of the building construction site, and the physical equipment architecture and construction site network of the BIMbased building construction site information management system are designed. The construction of this system provides a solution for applying BIM technology to the whole construction site and accelerates the information management process of the whole process of the construction industry.

\section{Data Availability}

The data used to support the findings of this study are available from the corresponding author upon request.

\section{Conflicts of Interest}

The author declares that they have no known competing financial interests or personal relationships that could have appeared to influence the work reported in this paper.

\section{References}

[1] V. Freschi and E. Lattanzi, "A study on the impact of packet length on communication in low power wireless sensor networks under interference," IEEE Internet of Things Journal, vol. 6, no. 2, pp. 3820-3830, 2019.

[2] R. Chéour, S. Khriji, D. El Houssaini, M. Baklouti, M. Abid, and O. Kanoun, "Recent trends of FPGA used for low-power wireless sensor network," IEEE Aerospace and Electronic Systems Magazine, vol. 34, no. 10, pp. 28-38, 2019.

[3] S. Bin and G. Sun, "Optimal energy resources allocation method of wireless sensor networks for intelligent railway systems," Sensors, vol. 20, no. 2, p. 482, 2020.

[4] K. Guleria and A. K. Verma, "Comprehensive review for energy efficient hierarchical routing protocols on wireless sensor networks," Wireless Networks, vol. 25, no. 3, pp. 1159-1183, 2019.

[5] H. H. Mahmoud, H. M. ElAttar, A. Saafan, and H. ElBadawy, "Optimal operational parameters for 5G energy harvesting cognitive wireless sensor networks," IETE Technical Review, vol. 34, no. sup1, pp. 62-72, 2017.

[6] H. Keshmiri and H. Bakhshi, "A new 2-phase optimizationbased guaranteed connected target coverage for wireless sensor networks," IEEE Sensors Journal, vol. 20, no. 13, pp. 7472 7486,2020 .

[7] I. Mandourarakis and E. Koutroulis, "Unified system-and circuit-level optimization of res-based power-supply systems for the nodes of wireless sensor networks," IEEE Transactions on Industrial Informatics, vol. 14, no. 2, pp. 598-607, 2018.

[8] S. Rani, S. H. Ahmed, and R. Rastogi, "Dynamic clustering approach based on wireless sensor networks genetic algorithm for IoT applications," Wireless Networks, vol. 26, no. 4, pp. 2307-2316, 2020. 
[9] X. Song, Y. Gong, D. Jin, and Q. Li, "Nodes deployment optimization algorithm based on improved evidence theory of underwater wireless sensor networks," Photonic Network Communications, vol. 37, no. 2, pp. 224-232, 2019.

[10] G. Shabbir, A. Akram, M. M. Iqbal, S. Jabbar, M. Alfawair, and J. Chaudhry, "Network performance enhancement of multisink enabled low power lossy networks in SDN based Internet of Things," International Journal of Parallel Programming, vol. 48, no. 2, pp. 367-398, 2020.

[11] K. Vijayalakshmi and P. Anandan, "A multi objective Tabu particle swarm optimization for effective cluster head selection in WSN," Cluster Computing, vol. 22, no. S5, pp. 12275-12282, 2019.

[12] X. Cao, S. Madria, and T. Hara, "Multi-model Z-compression for high speed data streaming and low-power wireless sensor networks," Distributed and Parallel Databases, vol. 38, no. 1, pp. 153-191, 2020.

[13] F. Gara, L. B. Saad, R. B. Ayed, and B. Tourancheau, "A new scheme for RPL to handle mobility in wireless sensor networks," International Journal of Ad Hoc and Ubiquitous Computing, vol. 30, no. 3, pp. 173-186, 2019.

[14] A. Karaagac, E. De Poorter, and J. Hoebeke, "In-band network telemetry in industrial wireless sensor networks," IEEE Transactions on Network and Service Management, vol. 17, no. 1, pp. 517-531, 2020.

[15] S. Bin and G. Sun, "Matrix factorization recommendation algorithm based on multiple social relationships," Mathematical Problems in Engineering, vol. 2021, 8 pages, 2021.

[16] J. Joy Winston and M. Nivas, "Optimal energy efficient connected coverage wireless sensor networks," Annals of the Romanian Society for Cell Biology, vol. 25, no. 6, pp. 2081220817, 2021.

[17] H. Wang, K. Li, and W. Pedrycz, "An elite hybrid metaheuristic optimization algorithm for maximizing wireless sensor networks lifetime with a sink node," IEEE Sensors Journal, vol. 20, no. 10, pp. 5634-5649, 2020.

[18] G. Sun, C. C. Chen, and S. Bin, "Study of cascading failure in multisubnet composite complex networks," Symmetry, vol. 13, no. 3, p. 523, 2021.

[19] X. Liu, Z. Sheng, C. Yin, F. Ali, and D. Roggen, "Performance analysis of routing protocol for low power and lossy networks (RPL) in large scale networks," IEEE Internet of Things Journal, vol. 4, no. 6, pp. 2172-2185, 2017.

[20] A. Pegatoquet, T. N. Le, and M. Magno, "A wake-up radiobased MAC protocol for autonomous wireless sensor networks," IEEE/ACM Transactions on Networking, vol. 27, no. 1, pp. 56-70, 2019. 